\title{
Correlation Effects and Spin Dependent Transport in Carbon Nanostructures
}

\author{
S. LIPIŃSKI*, D. KRYCHOWSKI \\ Institute of Molecular Physics, Polish Academy of Sciences \\ M. Smoluchowskiego 17, 60-179 Poznań, Poland
}

\begin{abstract}
The impact of symmetry breaking perturbations on the spin dependent transport through carbon nanotube quantum dots in the Kondo regime is discussed. The proposals of spin filter, spin valve and spin battery are presented.
\end{abstract}

DOI: 10.12693/APhysPolA.126.190

PACS: 72.10.Fk; 73.63.Fg; 73.63.Kv; 85.75.-d

\section{Introduction}

According to many predictions on the evolution of microprocessor technology [1], around two thousand twenty the limit of a single atom per bit will be reached and it is the range where devices will be governed by purely quantum mechanical laws. But before this ultimate atomic limit is reached, there is earlier the range of ten atoms per bit, and already at this scale silicon technology gets in trouble. There are many reasons for the predicted collapse of silicon technology, the most severe limitation comes from thermodynamics. A by-product of computing operation is the excess heat, the relative contribution of dissipation increases with the scale of integration [2]. Therefore without improvement of the energy efficiency, such computers will just melt during processing.

It is believed that the next revolution in electronics would be based on molecules [3] and in particular on carbon systems [4]. In contrast to silicon-based devices, the molecular can successfully cope with the more and more demanding miniaturization requirement without worsening their multi-functional properties.

Conventional electronics has ignored the spin of electron. Recently very rapidly is developing a new branch of nanotechnology - spintronics, which exploits this degree of freedom. This field brings memory and logic functionalities on the same chip. Spin - the ultimate logic bit is an attractive degree of freedom, because the energy scale relevant for its typical dynamics $(10-100 \mathrm{meV})$ is order of magnitudes smaller than that involved in manipulating the electron charge in standard transistors $(1 \mathrm{eV})$. This translates in devices exhibiting ultra-low power consumption and high speed [5]. Carbon systems are particularly attractive for spintronic applications due to the weak spin-orbit and hyperfine interactions [6]. The corresponding spin-diffusion lengths are of order of several hundreds nms.

In order to move towards quantum information technologies, spintronics at the single spin level is required.

${ }^{*}$ corresponding author; e-mail: lipinski@ifmpan.poznan.pl
When we connect a single nano-object to two magnetic electrodes, the spin dependent transport is predicted to interplay with single electron physics, i.e. with Coulomb blockade in the case of weak coupling [7] or with the Kondo effect in the case of stronger coupling between the nano-object and electrodes [8].

We will present here a very brief review of our recent study of exotic Kondo effect in carbon nanotube quantum dot (CNT-QD) and propositions how this phenomenon can be exploited in spintronics. Detailed reviews can be found in Refs. [9, 10].

\section{Spin-orbital Kondo effect}

Figure 1a presents schematic view of carbon nanotube quantum dot (CNT-QD). Such a dot is formed when electrons are confined to a small region within a carbon nanotube [7]. Experimentally it is accomplished by laying a CNT on a silicon dioxide surface, sitting on a doped silicon waver. The silicon wafer serves as the gate electrode.

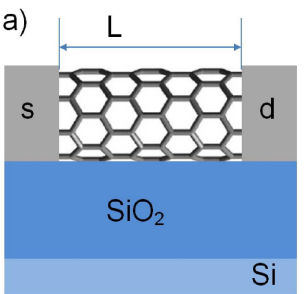

$\mathrm{s}=\mathrm{N}(\uparrow) \mathrm{d}=\mathrm{N}(\uparrow[\downarrow])$

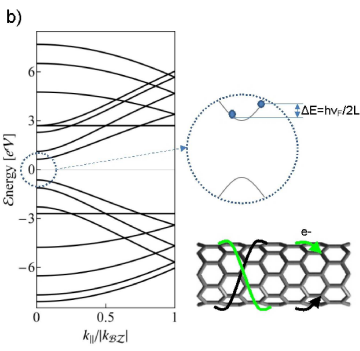

Fig. 1. a) Side view of CNT-QD with paramagnetic electrodes $(\mathrm{N})$ or with ferromagnetic electrodes $((\uparrow)$ or $(\downarrow)$ - spin valve). b) Band structure of semiconducting zigzag CNT C $(4,0)$. Zoom view illustrates quantum confinement quantization of energy levels and bottom right picture visualizes orbital degeneracy - clockwise and anticlockwise orbital motion.

Metallic leads can then be laid over the nanotube in order to connect the dot up to an electrical circuit. When the resistance of the two tunnel barriers becomes comparable to, or is larger than the quantum resistance $\left(\mathcal{R}_{Q}=h / 2 e^{2}\right)$, the island becomes strongly separated. A finite length $L$ between the electrodes results in quantized energy levels $\Delta E \approx h v_{F} / 2 L$, where $v_{F}$ denotes Fermi 
velocity (Fig. 1b). In an ideal semiconducting nanotube, sets of four electronic states can be grouped together into a shell. All four states are degenerate, with two choices for spin and two for orbital.
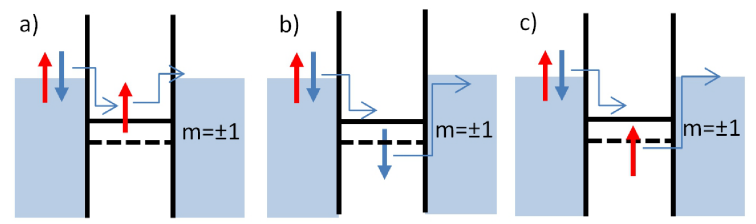

Fig. 2. Cotunneling processes in SU(4) quantum dot: a) spin-flip fluctuation, b) orbital fluctuation, c) spinorbital fluctuation.

The orbital degeneracy can be intuitively viewed to originate from two equivalent ways electrons can circle the graphene cylinder, that is clockwise and anticlockwise (Fig. 1b). Kondo effect is observed at low temperatures in the intermediate coupling range i.e. when tunnel induced broadening of energy levels $\Gamma$ is smaller than charging energy $\Gamma \leq E_{C}$. In this regime the role of higher order tunneling processes increases. The Kondo spin screening and the associated formation of manybody resonance, which leads to the enhanced transmission at the Fermi level, results from virtual spin flips at the dot, caused by tunneling processes off the dot with a given spin followed by tunneling of electron of opposite spin on the dot. Adding many spin-flips processes of higher order coherently, the spin-flip rate diverges, establishing the characteristic temperature - energy scale of quasiparticle resonance. Kondo temperature sets the temperature, respectively the voltage or magnetic field scale above which the Kondo resonance is suppressed. The Kondo effect can also occur by replacing the spin by orbital [11] or charge [12] degrees of freedom. The necessary condition for the occurrence of this effect is the same degeneracy of the states in the electrodes and in the QD and conservation of spin or pseudospin in tunneling processes. For the two-fold degenerate states the allowed symmetry operations are rotations in spin space $(\mathrm{SU}(2))$. Spin and orbital degeneracies can also occur simultaneously, leading to highly symmetric Kondo state (SU(4)). Most spectacular evidence of this phenomena has been reported by Jarillo-Herrero et al. for carbon nanotubes [13]. SU(4) group characterizes the rotational invariance in spin and orbital space. The simultaneous screening of orbital and spin degrees is caused by tunneling processes, causing spin, orbital pseudospin and spin-orbital fluctuations (Fig. 2).

In this case orbital psudospins play exactly the same role as spins. The following numerical results are presented, taking $|e|=g=\mu_{B}=k_{B}=h=1$ and choosing $\Gamma$ as the energy unit $(\Gamma=1 \mathrm{meV})$, and $\Gamma_{\alpha}$ is the coupling to the $\alpha$-electrode $\left(2 \Gamma=\Gamma_{L}+\Gamma_{R}\right)$. Figure 3 a compares linear conductances and transmissions for $\mathrm{SU}(2)$ and SU(4) symmetries in the infinite Coulomb interaction limit $U \rightarrow \infty$. For deep dot level positions the unitary
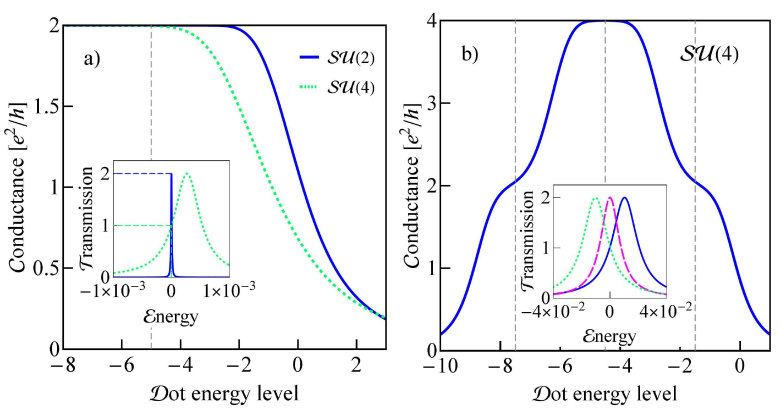

Fig. 3. a) Linear conductances of $\mathrm{SU}(4)$ and $\mathrm{SU}(2)$ Kondo dots vs. dot energy level for $U \rightarrow \infty$. Inset compares corresponding transmissions for both symmetries $\left(\varepsilon_{0}=-5\right)$. b) Conductance of SU(4) CNT-QD for $U=3$. Inset shows the corresponding transmissions from right to left for $N=1,2,3$ valleys (slave boson results - Kotliar-Ruckenstein SBMFA).

limit of total conductance is reached for both symmetries, and thus from conductance measurements alone one can not distinguish in the odd electron valleys between these two symmetries. The difference can be inferred from measurements in magnetic field or from analysis of shot noise [10] or, as it is presented in Fig. 3b, by moving to the two-electron valley range. The spin-orbital many body peak is also much broader than spin fluctuation $\mathrm{SU}(2)$ peak, which means exponential enhancement of Kondo temperature. The use of single wall carbon nanotubes has pushed the Kondo temperatures to the range of several K [13], in graphene nanostructures even higher Kondo temperatures are expected [14]. SU(4) transmission in $N=1$ ( $N$ denotes electron occupation) valley shows a peak slightly shifted from the Fermi energy, it is pinned at $\mathcal{E} \sim T_{K}$. For $N=3$ (single hole) the resonance is similarly shifted from the Fermi level, but towards the negative energy values. We also present in Fig. 3b conductance of CNT-QD in the Kondo range for finite U obtained with the use of two-orbital Anderson model. The pronounced enhancements of conductance are observed not only in odd electron valleys, but also in two-electron valley. In the latter case all six degenerate two electron states participate in the formation of $\mathrm{SU}(4)$ Kondo resonance [15]. The results are in qualitative agreement with experimental data of Makarovski et al. [15], but we have assumed symmetric coupling to the leads, whereas experimental devices were coupled highly asymmetrically. In the following discussion we will analyze an impact of symmetry breaking perturbations on the spin dependent transport.

\subsection{Kondo spin valve}

The prototype of all spin devices is the spin valve on which the read heads of computer drives and magnetic random access memories are based. It consists of a non-magnetic material sandwiched between two ferromagnetic electrodes. The flow of carriers through a spin valve is determined by the direction of their spin (up or down), relative to the magnetic polarization of the 
device's electrodes. The relative orientation of polarizations can be tuned by an external magnetic field. The difference in resistance between antiparallel and parallel states is known as its magnetoresistance (MR). In carbon based spintronic device the role of spacer is played by graphene, carbon nanotube or fullerene. Figure 1a can serve as a schematic presentation of CNT spin valve when electrodes are ferromagnetic.
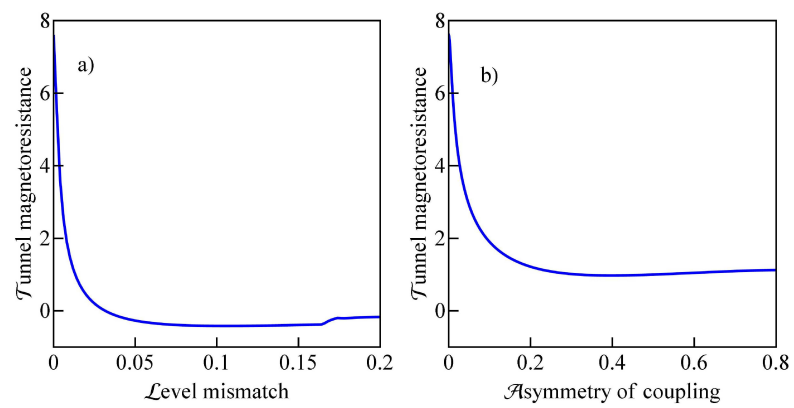

Fig. 4. Linear TMR as a function of a) orbital level mismatch, b) asymmetry of couplings (Equation of motion (EOM) calculations with $\varepsilon_{0}=-6, U=15$, polarization of electrodes - relative difference of densities of states for up and down spins at $\mathcal{E}_{F}$, is taken $P=0.6$ ).

The first observed tunneling magnetoresistance signal (TMR) in molecular system was reported for multiwall carbon nanotube contacted to polycrystalline Co electrodes [16]. Recently we have shown, that for CNT-QDs coupled to ferromagnetic electrodes, the giant values of TMR are expected in Kondo regime for some ranges of gate voltages [10]. The role of polarization of electrodes is twofold: it makes the tunneling processes spin dependent and it introduces an effective exchange field via spin-dependent charge fluctuations [17]. By changing the gate voltage one can move the system to different ranges of charge fluctuations, which reflects in the change not only of the value, but also of the sign of effective exchange splitting. This in turn results in drastic changes of TMR. Control by electric means is currently an important challenge for spintronics, because in contrast to an applied magnetic field, electric field acts rapidly and allows very localized addressing. The question arises how robust is TMR against disturbances. Due to disorder or deformations the full spin-orbital degeneracy of nanotube dots is rather an exception than the rule. Two examples of the impact of nonmagnetic perturbations on TMR are shown in Fig. 4, the effect of orbital level mismatch $\Delta_{o r b}=\varepsilon_{2}-\varepsilon_{1}$, and influence of asymmetry of coupling of the dot to the electrodes $\left(1-\Gamma_{L} / \Gamma_{R}\right)$. It is seen that the record values of magnetoresistance are achieved for the full symmetric case.

\subsection{Kondo spin filter}

Spin filter is a device that filters electrons by their spin orientation. In quantum information technology spin filters can be used for initialization and readout of spin quantum bits [18]. For systems with broken symmetry the increase of conductance can be achieved by field induced recovery of degeneracy, allowing for restoration of Kondo effect. Here we discuss tuning of spin polarized orbitally mismatched states by magnetic field. If the states are chosen from the same energy shell, the filter would operate in the low field range, for orbital mismatch $\Delta_{\text {orb }}=0.01 \mathrm{meV}$ the required filtering fields are of order of $10 \mathrm{mT}$. Orbital level mismatch occurs in nanotubes with torsional deformation. Axial magnetic field might recover the orbital degeneracy either within the same spin sector $\left(h_{0}=\frac{\Delta_{\text {orb }}}{2 \mu_{\text {orb }}}, \mu_{\text {orb }}\right.$ - orbital magnetic moment, typically $\mu_{\text {orb }} \sim 10 \mu_{B}$ ) or with mixing the spin channels $\left(h_{1,2}=\frac{\Delta_{\text {orb }}}{2\left(\mu_{\text {orb }} \mp 1\right)}\right)$ (see Fig. 5a).
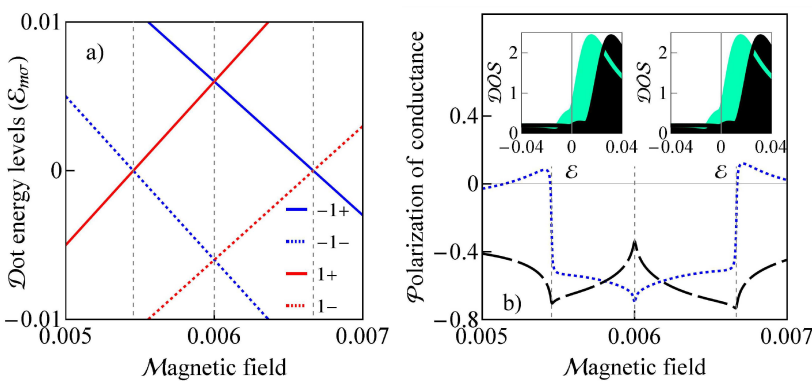

Fig. 5. a) Illustration of recovery of orbital degeneracy by axial magnetic field $\left(\Delta_{\text {orb }}=0.012, \mu_{\text {orb }}=10 \mu_{B}\right)$. b) Polarization of conductance vs. axial magnetic field: dotted line is for Kondo regime $\left(\varepsilon_{0}=-6\right)$ and broken line corresponds to MV range $\left(\varepsilon_{0}=-4\right)$. Insets show the spin resolved densities of states for $h_{0}$, left for Kondo regime and right for MV case. Dark and grey/green filled curves correspond to spin up and spin down respectively. (EOM results with $U=15$ ).

In the former case spin up and spin down Kondo resonances emerge due to orbital fluctuations and in the latter, single Kondo resonance is formed due to spin orbital fluctuations. Apart from the many body resonances, resulting from fluctuations between degenerate states, the satellites, reflecting fluctuations between nondegenerate spin-orbital states, are also visible (insets of Fig. 5b). We illustrate the field dependence of polarizations for two representative gate voltages, corresponding to Kondo range of the dot and to the mixed valence range (MV). The Kondo like resonances in the Kondo regime are pinned at the Fermi level, whereas in MV state they are shifted above (Fig. 5b). The absolute value of polarization is maximized at $h_{0}$ in the Kondo range, when Kondo peaks for both spin channels cross the Fermi level. In the MV regime not the maxima but, the tails of the resonances cross $\mathcal{E}_{F}$ in this field. The observed gate dependence of polarization for the fixed magnetic field opens a path for electric field control of spin polarization.

\subsection{Kondo spin battery}

Recently, there has been an increasing interest in generation of pure spin current without an accompanying 
charge current [19]. The idea is that when spin-up electrons move to one direction while an equal number of spin-down electrons move to the opposite direction, the net charge current $\mathcal{I}^{\mathcal{C}}=e\left(\mathcal{I}_{+}+\mathcal{I}_{-}\right)$vanishes and a finite spin current $\mathcal{I}^{\mathcal{S}}=h / 2\left(\mathcal{I}_{+}-\mathcal{I}_{-}\right)$emerges. To be able to generate and control spin currents is one of the challenges of spintronics.
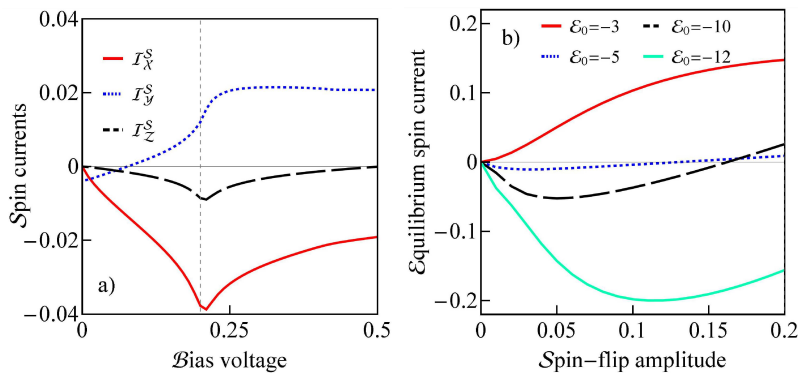

Fig. 6. a) Spin currents of CNT-QD for spin flip amplitude $\mathcal{R}=0.1$. b) Equilibrium spin current $\mathcal{I}_{y}^{\mathcal{S}}$ vs. spin-flip amplitude for several values of dot level (EOM, $U=15, P=0.6)$.

The attractive attribute of spin current is that it is associated with a flow of angular momentum, which is a vector quantity. This feature allows information to be sent across nanoscopic structures. As opposed to charge current a spin current is invariant under time reversal. This property determines the low dissipative or even dissipativeless spin transport. The present proposal presents gate controllable spin-battery based on CNTQD coupled to ferromagnetic electrodes with different magnetizations. In the following we consider antiparallely (AP) aligned ferromagnetic leads as illustrated in Fig. 1a. To allow the device to generate equilibrium spin currents we introduce real spin-flip processes at the dot, which mix the spin channels. They are represented by a perturbation $\mathcal{H}^{\prime}=\sum_{m=1(2)} \mathcal{R}\left(d_{m+}^{+} d_{m-}+h . c\right)$. Spin flips may be caused e.g., by transverse component of a local magnetic field. These processes are assumed to be coherent, in the sense that spin-flip strength $\mathcal{R}$ involves reversible transitions. The impact of Kondo effect on the spin currents is of special interest, since it provides also spin-flip processes (cotunneling mechanism). Real spin flip perturbation mixes the spin channels and therefore, beyond the longitudinal $\mathcal{I}_{z}^{\mathcal{S}}$ spin current also transverse currents appear for finite bias (Fig. 6a). But even for vanishing bias not all components of spin current vanish. Perturbation $\mathcal{H}^{\prime}$ is equivalent to operation of magnetic field in $x$ direction and thus the spin torque acting on spin aligned along $z$ direction acts along $y$ direction. Due to the opposite spin polarizations of the electrodes, the imbalance between the spin orientations in $y$ direction for left and right moving carriers is induced. This results in equilibrium $\mathcal{I}_{y}^{\mathcal{S}}$ component of spin current $(E S C)$. As it is seen from Fig. 6b, $E S C$ is controllable by gate voltage (different values of $\varepsilon_{0}$ ) or spin-flip amplitude (magnetic field), both these factors influence Kondo resonance. For large values of $\mathcal{R}$ the three peak structure of many-body resonances is expected for AP configuration, with satellites located roughly at $\mathcal{E} \approx T_{K} \pm 2 \mathcal{R}$. The satellites reflect in bias dependence of spin currents as maxima, minima or rapid changes for corresponding voltages (Fig. 6a). The described system would also generate $E S C$ if only one ferromagnetic electrode was attached to the dot with spin flips, and such a system can be viewed as spin battery [19].

\section{Acknowledgments}

This work was supported by the Polish Ministry of Science and Higher Education as a research Project No. N N202 199239 for years 2010-2013.

\section{References}

[1] G.E. Moore, Electronics 38, 114 (1965).

[2] N.S. Kim, T. Austin, D. Blaauw, T. Mudge, K. Flautner, J.S. Hu, M.J. Irwin, M. Kandemir, V. Narayanan, Computer 36, 68 (2003).

[3] J.C. Cuevas, E. Scheer, Molecular Electronics - An Introduction to Theory and Experiment, World Scientific Publishing Company, 2010.

[4] P. Avouris, Z. Chen, V. Perebeinos, Nature Nanotechnology 2, 605 (2007).

[5] W.J.M. Naber, S. Faez, W.G. van der Viel, J. Phys. D: Appl. Phys. 40, R205 (2007).

[6] A. Cottet, T. Kontos, S. Sahoo, H.T. Man, M.S. Choi, W. Belzig, C. Bruder, A.F. Morpurgo, C. Schönenberger, Semicond. Sci. Technol. 21, S78 (2006).

[7] S. Sapmaz, P. Jarillo-Herrero, L.P. Kouwenhoven, H.S.J. van der Zant, Semicond. Sci. Technol. 21, S52 (2006).

[8] L. Kouwenhoven, L. Glazman, Physics World 14, 33 (2001).

[9] S. Lipinski, D. Krychowski, phys. stat. sol. (b) 243 206 (2006).

[10] S. Lipinski, D. Krychowski, Phys. Rev. B 81, 115327 (2010).

[11] S. Sasaki, S. Amaha, N. Asakawa, M. Eto, S. Tarucha, Phys. Rev. Lett. 93, 017205 (2004).

[12] U. Wilhelm, J. Schmid, J. Weis, K. v. Klitzing, Physica E 14, 385 (2002).

[13] P. Jarillo-Herrero, J. Kong, H.S.J. van der Zant, C. Dekker, L.P. Kouvenhoven, S. De Franceschi, $\mathrm{Na}$ ture 434, 484 (2005).

[14] J.-H. Chen, L. Li, W.G. Cullen, E.D. Williams, M.S. Fuhrer, Nature Phys. 7, 535 (2011).

[15] A. Makarovski, A. Zhukov, J. Liu, G. Finkelstein, Phys. Rev. B 75, 241407(R) (2007).

[16] K. Tsukagoshi, B.W. Alphenaar, H. Ago, Nature 401, 572 (1999).

[17] J. Martinek, M. Sindel, L. Borda, J. Barnaś, R. Bulla, J. König, G. Schön, S. Maekawa, J. von Delft, Phys. Rev. B 72, 121302 (2005).

[18] D. Loss, D.P. DiVincenzo, Phys. Rev. A 57, 120 (1998).

[19] A. Brataas, Y. Tserkovnyak, G.E.W. Bauer, B.I. Halperin, Phys. Rev. B 66, 060404 (2002). 